\title{
THE BACTERICIDAL ACTION OF HUMAN SERUM ON HEMOLYTIC STREPTOCOCCI
}

I. Observations Made with Serum from Patients with Acute INFECTIONS AND FROM NORMAL INDIVIDUALS

By WILLIAM S. TILLETT, M.D.

(From the Biological Division, Department of Medicine, Johns Hopkins Medical School and Hospital, Baltimore)

(Received for publication, August 10, 1936)

The results presented in this communication concern the destructive action upon hemolytic streptococci of serum from patients who are acutely ill. Successive samples of serum have been obtained from representatives of a number of different kinds of infectious diseases, both during acute illness and following recovery, and determinations of bactericidal power have been made by the use of different strains of Streptococcus hemolyticus of the beta type. The several specimens. of sera have exhibited marked differences in streptococcidal potency which may be correlated with the condition of the patient at the time at which the blood was obtained. The results which are described in detail in this article were obtained with sera from twenty-five adult patients acutely ill with different infections. The list is as follows:

13 cases of pneumococcus pneumonia.

3 cases of acute respiratory infection of uncertain etiology (lung abscess, bronchitis?, pyopneumothorax).

1 case of tuberculous pleurisy with effusion.

2 cases of acute tonsillitis due to hemolytic streptococcus.

2 cases of meningococcus meningitis.

1 case of staphylococcus septicemia.

Total 25

3 cases of induced malaria.

The attempt was made to select individuals whose illness was not mild but might be expected to be reasonably short and thus afford the 
opportunity of making observations as rapid changes in the severity of the condition occurred. The patients with pneumonia or induced malaria were particularly favorable since recovery was usually abrupt and complete. All of the patients were severely or moderately severely ill and in most instances the temperature was high during the acute period. Data with respect to fever and other points are presented in Tables I and II.

Comparable tests were also made with sera from normal individuals; twenty healthy adults, in the third to fifth decades of life, were used for this purpose.

Studies directed toward an understanding of the nature of the reaction have also been in progress and the information, which has accrued from this phase of the investigation, will be given in a second article (1).

\section{Materials and Methods}

Serum.-Blood was obtained under sterile precautions. The serum was separated from the clot, usually within a few hours after coagulation had occurred. The specimens were always centrifuged twice in order to insure the removal of leucocytes, and then kept in the ice box until used. Tests were usually carried out on the day after serum was obtained.

Cultures.-18 hour cultures, freshly grown, were always employed. The culture medium consisted of plain meat infusion broth, containing 1 per cent neopeptone and 0.05 per cent dextrose. The $\mathrm{pH}$ was adjusted to 7.6, but no buffer was added.

Observations made with many different strains of hemolytic streptococci have revealed differences in susceptibility of the cultures to the bactericidal action of the test sera. Data concerning other characteristics of a large number of strains in relation to sensitiveness to streptococcidal action of sera, will be subsequently reported. In the phase of the subject dealt with in this paper, three selected strains were employed. They consisted of the most susceptible strain, the most resistant, and a third occupying an intermediate position.

1. Strain Sc.-Most sensitive strain. It was isolated Dec. 9, 1935, from the subcutaneous abscess of a 75 year old patient who died a few days later.

2. Strain Ba.-Most resistant strain. It was obtained Mar. 3, 1936, from the finger of a patient with erysipelas.

3. Strain Co.-Intermediate strain. It is an old laboratory strain which was recovered Mar. 4, 1931, from the blood of a patient with septicemia.

Before use, the relative abundance of growth of the three cultures was estimated on the basis of the turbidity of the broth. If-as rarely occurred-any difference was noted, the three cultures were reduced to the same degree of density by diluting with sterile broth those which were more turbid. 
Test.-1.0 cc. of serum in Wassermann tubes was inoculated with 1 platinum loopful of culture. The loop is $0.2 \mathrm{~cm}$. in diameter. A full, rounded drop of culture for inoculum was obtained by flipping the loop from the liquid while the culture tube was held on a slant. By serial dilutions and subculture in poured plates of blood agar, several observations were made on the number of viable organisms in an inoculum of this size. The estimates ranged from 800,000 to $1,500,000$, averaging approximately $1,000,000$. After inoculation, the tubes were placed in a water bath at $37.5^{\circ} \mathrm{C}$.

Subcultures were made as follows: (a) Serum before introducing organisms. These cultures were always sterile. (b) Serum-streptococcus mixture immediately after inoculation. $1000 \mathrm{~S}$ to $\infty$ number of colonies were present. (c) 6 hours after inoculation. (d) 24 hours after inoculation. (e) Occasionally 48 hours after inoculation. For subculture, a loopful of serum comparable in size to that used for inoculum of culture, was put into $10 \mathrm{cc}$. of melted agar containing $0.7 \mathrm{cc}$. of defibrinated rabbit blood. The tube was rapidly and vigorously rolled between the palms of the hands and plates were poured. The even distribution of colonies, after growth had taken place, indicated that the procedure afforded satisfactory mixing of serum in the melted agar.

The plates were inspected after 24 hours incubation. If less than 1000 colonies were present, they were incubated for another 24 hours before final reading. The number of colonies in the plates was used as an index of survival or death of streptococci. No attempt was made to differentiate between survival and additional multiplication. The grading of the number of organisms in the plates was as follows:

$\infty$ designates innumerable colonies after 24 hours incubation, with complete hemolysis of the whole plate.

1000 designates a number less than $\infty$, but so great that accurate counting was impractical. Small areas of unchanged blood were present in the plates after 24 hours incubation.

When colonies were less numerous, the plate was divided into fourths or eighths and the total was estimated in round numbers from the average of two or three sections. When the number was below 100 , all the colonies were counted.

The procedure which has just been described has been used, with various modifications, by numerous investigators. The two objections most frequently advanced against it as a measure of bactericidal action are based on: first, variations in the number of colonies in subculture due to differences in length of chain formation of streptococci, and second, the effect of agglutination by sera on the dispersion of organisms throughout the subculture. In the present experiments, the first of the criticisms is rendered invalid, since repeated microscopical examinations of the serum-streptococcus mixtures demonstrated that there was no correlation between chain formation and 
number of colonies. Special consideration has been given to the question of agglutination. Experiments bearing on this point will be described in the second article (1). At the present time it is sufficient to state that many of the sera have been tested with heat-killed organisms at $56^{\circ} \mathrm{C}$., and that no antistreptococcus agglutinins have been found.

Before proceeding with a description of the results obtained with sera from patients, data derived from tests made with normal sera will be given. Twenty different adults (sixteen males and four females) supplied specimens of blood. Each of the sera was tested with each of the three strains of hemolytic streptococcus. Repeated specimens were obtained from some of the normal individuals at intervals of several days and weeks. In view of the fact that the results were essentially the same in every instance, they are summarized as follows:

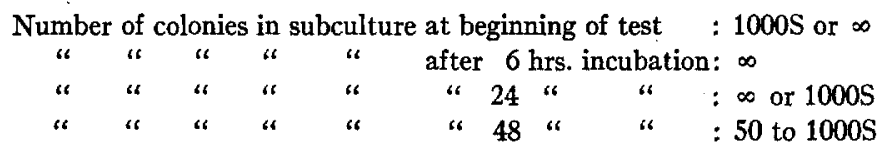

As the summary indicates, streptococci maintain approximately the same, or perhaps an increased, population during 24 hours incubation in normal human serum. With the experimental procedures which were used, such slight differences in the three strains as occurred were considered to be negligible. After incubation had lasted for 48 hours, however, there was a definite, but very variable decrease in the number of colonies on subculture of each of the strains. The reason for the late effect is not yet understood. It may represent a delayed and slowly acting process comparable to that exhibited by patients' sera, or it may be dependent upon the influence of extraneous factors such as partial evaporation of water from serum or the accumulation of metabolic products. Since the action of patients' sera occurred in the first 24 hours of incubation and since the late subculture contributed no additional information to the effect of patients' sera, only the observations of the first 24 hours have been analyzed. An explanation of the late effect of normal serum must await further investigation. For the purposes of the experiments with which this study is concerned, the significant finding is the absence of streptococcidal action by normal serum during the first 6 to 24 hours of incubation. In every test in which patients' sera were used, a similar test was carried out with normal serum, thus insuring an adequate control. 
TABLE I

Streptococcidal Action of Serum from Patients with Lobar Pneumonia (Pneumococcus)

Tested with Three Strains (Sc, Co, and Ba) of Hemolytic Streptococci

The number of colonies present in subcultures made at beginning of experiment was always $1000 S$ to $\infty$

\begin{tabular}{|c|c|c|c|c|c|c|c|c|c|}
\hline \multirow{2}{*}{ Patient } & \multirow{2}{*}{ Clinical course } & \multirow{2}{*}{$\begin{array}{l}\text { Day } \\
\text { of } \\
\text { dis- } \\
\text { ease }\end{array}$} & \multirow{2}{*}{$\begin{array}{l}\text { Tem- } \\
\text { pera- } \\
\text { ture }\end{array}$} & \multicolumn{2}{|c|}{$S c^{*}$} & \multicolumn{2}{|c|}{$\mathrm{Co}^{*}$} & \multicolumn{2}{|c|}{$\mathrm{Ba}^{*}$} \\
\hline & & & & $6 \mathrm{hrs.t}$ & $24 \mathrm{hrs} . \dagger$ & $6 \mathrm{hrs}$. & 24 hrs. & 5 hrs. & $4 \mathrm{hrs}$. \\
\hline M. F. & $\begin{array}{l}\text { Recovery 9th-10th } \\
\text { day. Pneumococ- } \\
\text { cus group IV }\end{array}$ & $\begin{array}{r}4 \\
7 \\
15 \\
23\end{array}$ & $\begin{array}{l}103.6 \\
103.6 \\
99.4 \\
99.8\end{array} \mid$ & \begin{tabular}{c|}
$-\neq$ \\
2 \\
$\infty$ \\
$\infty$
\end{tabular} & $\left|\begin{array}{c}- \\
- \\
1000 \mathrm{~S} \\
\infty\end{array}\right|$ & $\begin{array}{r}20 \\
2\end{array}$ & \begin{tabular}{l|}
- \\
- \\
$\infty$
\end{tabular} & $\begin{array}{l}\infty \\
\infty \\
\infty \\
\infty\end{array}$ & $\begin{array}{l}1000 \mathrm{~S} \\
\infty \\
1000 \mathrm{~S} \\
\infty\end{array}$ \\
\hline W. S. & $\begin{array}{l}\text { Recovery } 10 \text { th-11th } \\
\text { day. Pneumococ- } \\
\text { cus Type X }\end{array}$ & $\begin{array}{l}5 \\
13 \\
20\end{array}$ & $\begin{array}{r}104.6 \\
99.4 \\
99.0\end{array}$ & $\begin{array}{c}- \\
150 \\
\infty\end{array}$ & $\begin{array}{c}- \\
- \\
1000 \mathrm{~S}\end{array}$ & $\begin{array}{l}\infty \\
\infty\end{array}$ & $\begin{array}{l}- \\
\infty \\
\infty\end{array}$ & $\begin{array}{l}1000 \\
\infty \\
\infty\end{array}$ & $\begin{array}{ll} & 1 \\
\infty & \\
\infty & \end{array}$ \\
\hline G. J. & $\begin{array}{l}\text { Marked improvement } \\
\text { 10th day. Low } \\
\text { fever until dis- } \\
\text { charge } 34 \text { th day }\end{array}$ & 13 & $\left|\begin{array}{l}101.2 \\
100.0\end{array}\right|$ & - & $\begin{array}{c}- \\
1000 \mathrm{~S}\end{array}$ & $\begin{array}{l}350 \\
\infty\end{array}$ & - & $\infty$ & $\begin{array}{l}1000 \mathrm{~S} \\
1000 \mathrm{~S}\end{array}$ \\
\hline R. C. & $\begin{array}{l}\text { Rapid recovery 2nd- } \\
\text { 3rd day. Pneumo- } \\
\text { coccus group IV }\end{array}$ & $\begin{array}{r}1-2 \\
5\end{array}$ & $\begin{array}{r}102.8 \\
98.6\end{array}$ & - & - & $\infty$ & $\begin{array}{c}- \\
1000 \mathrm{~S}\end{array}$ & $\infty$ & $\infty$ \\
\hline W. T. & $\begin{array}{l}\text { Crisis 9th day. } \\
\text { Pneumococcus } \\
\text { group IV }\end{array}$ & $\begin{array}{r}3 \\
7 \\
14\end{array}$ & $\left|\begin{array}{l}104.0 \\
105.4 \\
98.8\end{array}\right|$ & $\begin{array}{r}15 \\
90 \\
\infty\end{array}$ & - & $\begin{array}{r}22 \\
150 \\
\infty\end{array}$ & - & $1000 \mathrm{~S}$ & 800 \\
\hline C. $\mathrm{S}$. & $\begin{array}{l}\text { Gradual improve- } \\
\text { ment beginning 9th } \\
\text { day. Febrile until } \\
\text { 13th day. Pneu- } \\
\text { mococcus Type III }\end{array}$ & $\begin{array}{l}15 \\
20 \\
28\end{array}$ & $\left|\begin{array}{l}105.2 \\
101.8 \\
99.4 \\
99.2 \\
98.6\end{array}\right|$ & $\begin{array}{r}22 \\
240 \\
\infty\end{array}$ & -2 & $\begin{array}{l}- \\
- \\
\infty \\
\infty \\
\infty\end{array}$ & $\begin{array}{ll}\infty & \\
& 2\end{array}$ & $\begin{array}{c}1000 \mathrm{~S} \\
\infty \\
\infty\end{array}$ & $\begin{array}{l}200 \\
\infty\end{array}$ \\
\hline O. T. & $\begin{array}{l}\text { Bronchopneumonia } \\
\text { with low grade fe- } \\
\text { ver until } 23 \mathrm{rd} \text { day }\end{array}$ & 28 & $\left|\begin{array}{c}101.6 \\
99.8\end{array}\right|$ & $\infty$ & $\begin{array}{c}- \\
1000 S\end{array}$ & $\begin{array}{c}1000 S \\
\infty\end{array}$ & - & $\infty$ & $\begin{array}{l}350 \\
\infty\end{array}$ \\
\hline
\end{tabular}

* Designates strain employed in experiments.

† Hours indicate length of incubation before subculture was made.

$¥$ The sign ( - ) indicates no growth in subculture. The numerals indicate number of colonies in subcultures. $1000 \mathrm{~S}$ and $\infty$ are approximate estimations when actual counts were impractical. 
TABLE I-Concluded

\begin{tabular}{|c|c|c|c|c|c|c|c|c|c|}
\hline \multirow{2}{*}{ Patient } & \multirow{2}{*}{ Clinical course } & \multirow{2}{*}{$\begin{array}{l}\text { Day } \\
\text { of } \\
\text { dis- } \\
\text { ease }\end{array}$} & \multirow{2}{*}{$\begin{array}{l}\text { Tem- } \\
\text { pera- } \\
\text { ture }\end{array}$} & \multicolumn{2}{|c|}{$\mathbf{S c}^{*}$} & \multicolumn{2}{|c|}{$\mathrm{Co}^{*}$} & \multicolumn{2}{|c|}{$\mathbf{B a}^{*}$} \\
\hline & & & & $6 \mathrm{hrs.t}$ & 24 hrs.t & $6 \mathrm{hrs}$. & $24 \mathrm{hrs}$ & $6 \mathrm{hrs}$. & $24 \mathrm{hrs}$. \\
\hline F. R. & $\begin{array}{l}\text { Pneumococcus Type } \\
\text { I. Serum-treated. } \\
\text { Marked improve- } \\
\text { ment 4th day. } \\
\text { Low fever until } \\
\text { 14th day }\end{array}$ & $\begin{array}{r}3 \\
5 \\
15 \\
22\end{array}$ & $\begin{array}{l}104.0 \\
99.0 \\
99.2 \\
99.0\end{array}$ & $\infty_{\infty}^{5}$ & $\begin{array}{l}- \\
- \\
\infty\end{array}$ & $\begin{array}{l}28 \\
\infty \\
\infty\end{array}$ & $\begin{array}{c}- \\
- \\
1000 S \\
\infty\end{array}$ & $\begin{array}{c}1000 \mathrm{~S} \\
\infty \\
\infty \\
\infty\end{array}$ & $\begin{array}{l}55 \\
10005 \\
\infty \\
\infty\end{array}$ \\
\hline J. M. & $\begin{array}{l}\text { Pneumococcus Type } \\
\text { I. Serum-treated. } \\
\text { Marked improve- } \\
\text { ment 6th day. } \\
\text { Low fever until } \\
12 \text { th day }\end{array}$ & $\begin{array}{r}4 \\
8 \\
8 \\
13\end{array}$ & $\begin{array}{r}104.0 \\
101.0 \\
99.8\end{array}$ & ${ }_{\infty}^{71}$ & ${ }_{\infty}^{-}$ & $\begin{array}{l}114 \\
\infty \\
\infty\end{array}$ & $\begin{array}{c}3 \\
\infty \\
1000 \mathrm{~S}\end{array}$ & $\begin{array}{c}1000 \mathrm{~S} \\
\infty \\
\infty\end{array}$ & $\begin{array}{c}1000 \mathrm{~S} \\
\infty \\
1000 \mathrm{~S}\end{array}$ \\
\hline A. $\mathrm{F}$. & $\begin{array}{l}\text { Pneumococcus Type } \\
\text { II. Serum- } \\
\text { treated. Definite } \\
\text { improvement 4th } \\
\text { day }\end{array}$ & $\begin{array}{r}3 \\
7 \\
7 \\
12 \\
15\end{array}$ & \begin{tabular}{|l|}
102.4 \\
99.6 \\
99.4 \\
99.6
\end{tabular} & $\begin{array}{c}\infty \\
1000 S \\
\infty\end{array}$ & $\begin{array}{r}15 \\
2 \\
2\end{array}$ & $\begin{array}{l}350 \\
\infty \\
\infty\end{array}$ & $\begin{array}{l}18 \\
\infty\end{array}$ & $1000 \mathrm{~S}$ & $\infty$ \\
\hline J. A. & $\begin{array}{l}\text { Pneumococcus Type } \\
\text { I. Serum-treated. } \\
\text { Operation for em- } \\
\text { pyema 36th day }\end{array}$ & $\begin{array}{r}4 \\
13 \\
21 \\
39 \\
47\end{array}$ & $\begin{array}{r}104.0 \\
101.8 \\
101.2 \\
100.0 \\
99.4\end{array}$ & $\begin{array}{l}- \\
400 \\
65 \\
\infty\end{array}$ & $\begin{array}{l}- \\
- \\
\infty^{5}\end{array}$ & $\begin{array}{l}- \\
175 \\
\infty \\
\infty\end{array}$ & \begin{tabular}{r|r|}
- & \\
& 1 \\
$\infty$ & 11 \\
$\infty$ &
\end{tabular} & $1000 \mathrm{~S}$ & $1000 \mathrm{~S}$ \\
\hline E. $P$. & $\begin{array}{l}\text { Pneumonia, septi- } \\
\text { cemia, endocar- } \\
\text { ditis. Pneumococ- } \\
\text { cus Type X. Died }\end{array}$ & $\begin{array}{l}11 ? \\
16 ? \\
18 ? \\
24 ? \\
33 ?\end{array}$ & $\begin{array}{l}104.8 \\
102.5 \\
102.4 \\
103.5 \\
103.0\end{array}$ & $\begin{array}{l}- \\
- \\
- \\
-\end{array}$ & $\begin{array}{l}- \\
- \\
- \\
-\end{array}$ & 3 & $\begin{array}{l}- \\
-\end{array}$ & $\begin{array}{r}280 \\
80 \\
400 \\
250 \\
300\end{array}$ & $\begin{array}{l}- \\
- \\
-\end{array}$ \\
\hline L. B. & $\begin{array}{l}\text { Pneumococcus group } \\
\text { IV. Serum ob- } \\
\text { tained } 6 \mathrm{hrs} \text {. before } \\
\text { death }\end{array}$ & 7 & 103.2 & 4 & - & 325 & 12 & & 250 \\
\hline $\begin{array}{c}20 \text { nor- } \\
\text { mal } \\
\text { adults }\end{array}$ & & & & $\infty$ & $\begin{array}{l}\infty \text { to } \\
1000 S\end{array}$ & $\infty$ & $\begin{array}{l}\infty \text { to } \\
1000 S\end{array}$ & $\infty$ & $\begin{array}{l}\infty \text { to } \\
1000 \mathrm{~S}\end{array}$ \\
\hline
\end{tabular}


The greatest number of observations were made with serum from patients having pneumococcus pneumonia. The results are presented in Table $I$. The table has been so arranged that, in the section assigned to each patient, a space separates, where possible, the period of acute illness from that of recovery or convalescence. The differences in the data recorded above and below this space reveal the changes in streptococcidal action of sera from each patient for each of the strains.

From Table I it may be seen that all of the sera taken during the acute stage of the illness were capable of destroying organisms of strain Sc, the most sensitive of the three. The number of colonies in subcultures made after 6 hours incubation decreased rapidly from the thousands present at the beginning of the experiment to less than fifteen or even none. The 24 hour subcultures were regularly sterile. With ten of the specimens, the whole cubic centimeter of serum was subcultured after 24 hours. No organisms were recovered in eight instances, and less than ten colonies grew from subcultures of the remaining two specimens. The complete destruction of all the bacterial cells is additional evidence that the phenomenon is a true bactericidal one.

The bactericidal action of these same sera on strain Co is also definite but, in some instances, proceeded at a slightly slower rate than on strain Sc. When strain $\mathrm{Ba}$ was used, evidence of the bactericidal action was obtained with the sera of only six of the thirteen patients; the remaining seven were as ineffective as normal sera against this strain. The difference in susceptibility of the three strains is also brought out by the results presented in Table II.

It may be seen, therefore, that lobar pneumonia is attended by the presence of a serological property which is antagonistic to the survival of an organism of a different species, and that the streptococcidal element was demonstrable in serum obtained within 48 hours of the beginning of pneumonia-patients R. C. and C. S., Table I-and persisted so long as the infectious process was active.

It may be further noted from the table that the effectiveness of the early samples of sera is no less striking than the absence or diminution of streptococcidal activity in specimens derived from patients after they had recovered. Within a week following the patient's critical recovery or marked improvement, the bactericidal property 
154 ACTION OF HUMAN SERUM ON STREPTOCOCCI. I

\begin{tabular}{|c|c|c|c|c|c|c|c|c|}
\hline 5 & & $\stackrel{n}{\pi}$ & 8 & 9. & 8 & & 8 & 8 융 8 \\
\hline D & $\begin{array}{l}8 \\
8\end{array}$ & 兽 & 8 & 용 & 8 & & 8 & $\begin{array}{lll}8 & 88\end{array}$ \\
\hline ن & ह్ & - & 8 & 11 & $1 \quad 1 \quad 0$ & $N$ & 1 & N స్ల 8 \\
\hline 旁 & 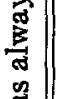 & 总 & $\stackrel{8}{0}$ & $1^{\infty}$ & สี่ & $\cong$ & ళ్లి & $\begin{array}{lll}8 & 8 & \\
8 & 8\end{array}$ \\
\hline 芯 & 范 & 量 & $1 \quad$ \&్ & $11 \quad$ \& & $\begin{array}{ll}N & 8 \\
& 8\end{array}$ & 1 & $N$ & $11^{-1} \delta$ \\
\hline $\begin{array}{l}3 \\
3 \\
3 \\
3\end{array}$ & 焉 & 离 & $\pi$ & $1^{2}$ & $\stackrel{\infty}{=} \stackrel{8}{\circ} 8$ & 1 & F & œ \\
\hline 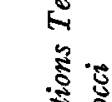 &. & 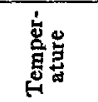 & $\mid \begin{array}{lll}4 & 0 \\
8 & 0 & 2\end{array}$ & 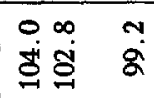 & 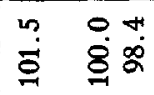 & & 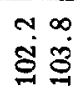 & 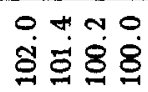 \\
\hline$\Xi$ & 4 & 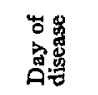 & $\infty \quad 2$ & No 尺ิ & $m \sim \pm$ & N & $2=$ & 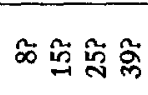 \\
\hline 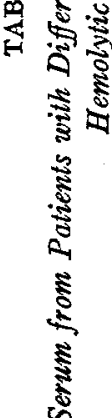 & 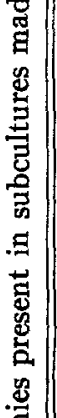 & 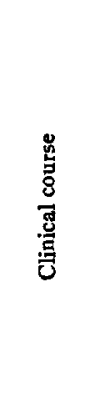 & 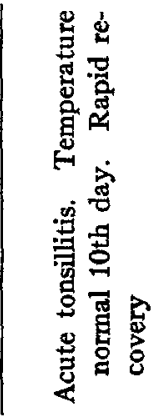 & 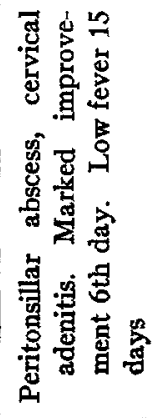 & 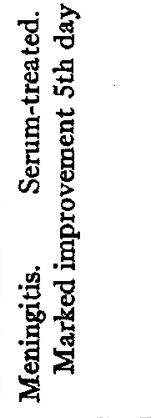 & 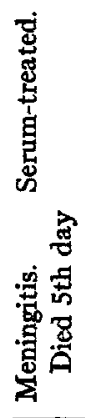 & 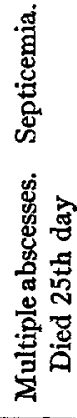 & 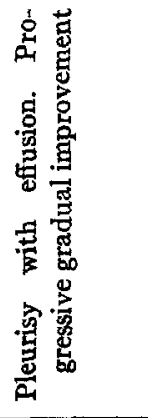 \\
\hline 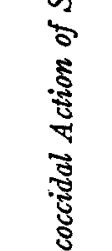 & 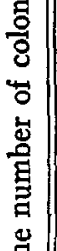 & 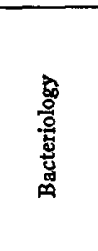 & 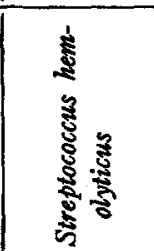 & 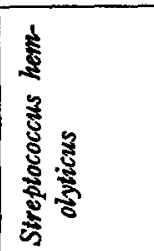 & 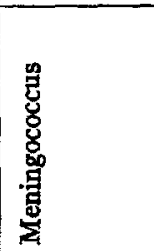 & 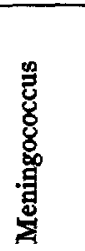 & 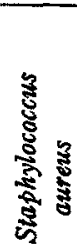 & 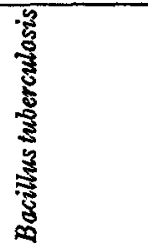 \\
\hline $\bar{n}$ & & 若 & $\begin{array}{l}\infty \\
\infty\end{array}$ & ن & $\dot{m}$ & $\dot{\vec{z}}$ & $\dot{0}$ & $\stackrel{i}{\dot{z}}$ \\
\hline
\end{tabular}


WILLIAM S. TILLETT

\begin{tabular}{|c|c|c|c|c|c|c|c|}
\hline ర్ర్రి 8 & $8 \quad 8$ & 8 & 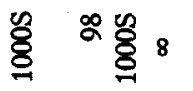 & & 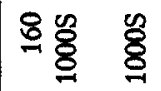 & 8 & $\begin{array}{l}2 \\
8 \\
8 \\
8\end{array}$ \\
\hline 8 & $8 \quad 8$ & 8 & $\begin{array}{lllll}8 & & 8 & 8 & 8\end{array}$ & & $\begin{array}{lll}8 & 8 & 8\end{array}$ & 8 & 8 \\
\hline & 18 & 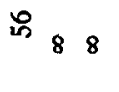 & $\overbrace{0}^{\infty} 1$ " & ళ్ & $\begin{array}{lll}1 & 8 & 8\end{array}$ & $1^{N} 8$ & $\begin{array}{l}8 \\
8 \\
8 \\
8\end{array}$ \\
\hline & 요 8 & สิ 88 & $8 \quad$ \&్ 8 है & 8 & $\begin{array}{llll}8 & 8 & 8\end{array}$ & $\begin{array}{lll}8 & 8 & 8\end{array}$ & 8 \\
\hline 1 ' & 18 & 0 in $\underset{9}{9}$ & $\overbrace{0}^{0} \quad 111$ & ${ }^{0} 8$ & $1^{*} \quad \overbrace{0}^{\mathscr{O}}$ & ${ }_{1}-8$ & 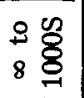 \\
\hline 188 & ธิ 8 & 아 88 & 8 近尔仝 & 88 & 용 8 & 옹스용 8 & 8 \\
\hline 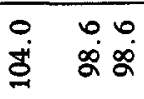 & 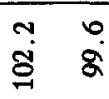 & $\begin{array}{l}0 \text { in } \\
8 \% 8 \\
8\end{array}$ & 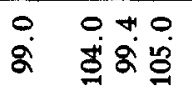 & \begin{tabular}{l}
$\sim \infty$ \\
\multirow{2}{*}{$: \infty$} \\
$\approx$
\end{tabular} & 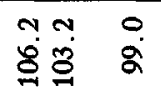 & 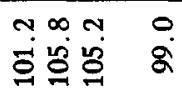 & \\
\hline$\infty \mp \mathbb{N}$ & 点 & 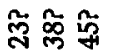 & $*=0$ & సని & $\# 0$ & 으묘 \& & \\
\hline 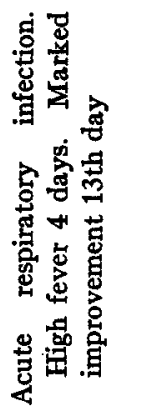 & 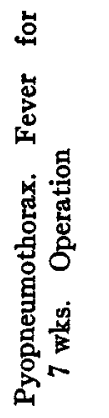 & 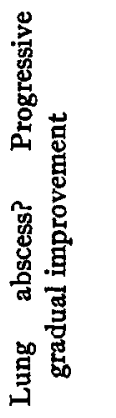 & 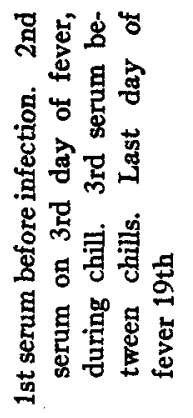 & & 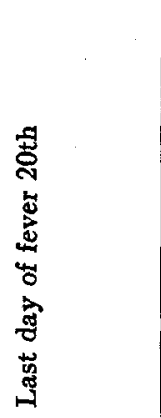 & 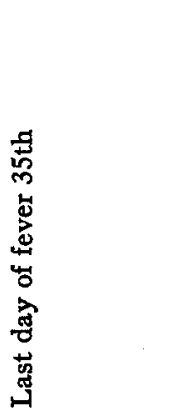 & \\
\hline $\begin{array}{l}\text { 烝 } \\
\text { 总 }\end{array}$ & 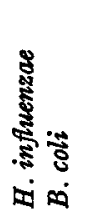 & 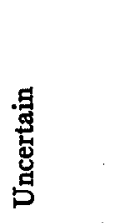 & 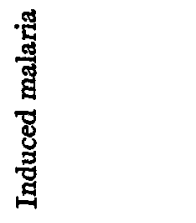 & & 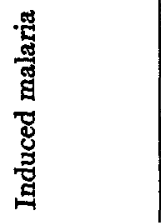 & $\begin{array}{l}\text { 恚 } \\
\text { 胥 } \\
\text { 总 } \\
\text { 总 } \\
\text { 总 }\end{array}$ & \\
\hline $\begin{array}{l}\dot{A} \\
\dot{H}\end{array}$ & $\dot{4}$ & ن & $\dot{m}$ & & E⿱宀 & $\begin{array}{l}\dot{\mathrm{i}} \\
\dot{0}\end{array}$ & 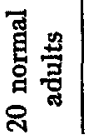 \\
\hline
\end{tabular}


for streptococci was either lost or definitely reduced in potency. In every instance but one, the last specimen of serum, obtained before discharge of the patients from the hospital, was indistinguishable from normal serum. One patient (J.A.) developed empyema. Repeated tests with his sera showed that the killing power persisted until after successful operative treatment.

Two patients, represented at the bottom of the table, died. Patient E. P. had pneumonia with septicemia and then developed bacterial (pneumococcus) endocarditis. The number of colonies of pneumococci in her blood cultures varied irregularly during the infection from none to several hundred. She died after an illness of $\sigma$ weeks. Her serum was continually one of the most potent with which these observations have been made. Patient L. B. had pneumonia with septicemia and died 7 days after onset. With the sample of her blood, obtained 6 hours before death, streptococcidal action was present to a high degree.

The results presented in Table II were obtained from similar tests made with sera from patients having acute diseases of different etiologies. Examples of infection with hemolytic streptococcus, meningococcus, staphylococcus, tubercle bacillus, and malarial parasites are presented. Although the number of individuals representative of each type of infection is few, the results are in every way comparable to those obtained with cases of lobar pneumonia due to pneumococcus. The streptococcidal property was high in specimens of serum taken during active disease and either disappeared within a few days after rapid recovery, or gradually diminished, when improvement was slow, as in the case of tuberculous pleurisy with effusion and that of pulmonary abscess.

From the data presented in Tables I and II, it may be concluded that the human serological property responsible for the destruction of hemolytic streptococci is evoked by different disease-producing agents and that its presence, which is transient, is determined by the activity of the infection. The difference in the rate and completeness of the destruction of the three strains is also noteworthy.

In reviewing the very extensive literature ${ }^{\mathfrak{l}}$ on the subject of the bactericidal action of blood, a few articles have been found which contain results somewhat

\footnotetext{
'Some idea of the extent of the literature concerning the bactericidal action of blood may be gained by referring to the article by Knorr (4); the text is followed by 24 pages of references with approximately 30 titles per page. The army of
} 
comparable to those just described. Dresel and Keller (2) reported results of observations on bactericidal action of serum from patients on anthrax bacillus (Anthrakocidin). They found that the serum from normal persons did not possess the property, but that it was present in serum from patients having a wide variety of acute or chronic diseases of an infectious or degenerative nature, such as tuberculosis, syphilis febrile or afebrile, pernicious anemia, leucemia, cirrhosis of liver, cardiac disease, and arterial sclerosis. They also noted that this property of the serum may be present for a considerable length of time after recovery or improvement. Trommsdorff (3) tested the killing power of serum from patients for staphylococci, typhoid and colon bacilli, and obtained evidence of bactericidal action. The latter two organisms were more easily destroyed than staphylococci. He noted that five normal sera were somewhat bacteriostatic but not bactericidal, and that among the sera from nine patients, six of whom had staphylococcus septicemia, five were listed as strong or moderately strong in bactericidin and four as weak or minimal. He tested further for alexin by determining the hemolysin titer of the sera and found some degree of parallelism between the two tests. He comments upon the fact that the alexin content of sera from both normal persons and patients may be variable in successive samples, and concludes that the quantitative determination of its presence in human sera is neither diagnostic nor prognostic.

The fact that extracts of leucocytes (leukin) have been found to be bactericidal has led some investigators to offer the suggestion that the bactericidin of serum may be derived from leucocytes. Among the patients included in this present study leucocytosis has often been present when the serum was highly bactericidal for streptococci. There were several exceptions, however, in cases with normal or leucopenic white blood cell count, but with sera markedly effective in streptococcidal activity. Consequently, a superficial correlation between number of leucocytes in the circulating blood and bactericidal potency of the serum offers no information concerning the suggested leucocytic origin of the streptococcidal properties.

All of the patients had fever, often $103^{\circ} \mathrm{F}$. or higher, and often con-

investigators in this field have used whole blood or defibrinated blood or serum or leucocytes. Blood has been obtained from many different animal species both normal and immunized. A wide variety of bacterial species have been employed. It is interesting to note that Topley and Wilson (5) state that "There are not many bacteriological problems which present quite so confusing and unsatisfactory a collection of data as do those concerned with the nature and mechanism of the bacteriolytic and bactericidal antibodies." In this article and the one that follows, citation of references has been limited to a few which seem to be directly pertinent to the subject matter under consideration. 
tinuous, during the acute period of sickness. Furthermore, the permanent disappearance of fever was closely followed by the loss of destructive action of serum. Accurate information concerning the significance of fever has not yet been obtained. However, patients receiving malaria by inoculation have afforded the opportunity of making observations which may be applicable to this aspect of the problem. Of the individuals with general paresis, who were admitted to the hospital for malarial therapy, data derived from three are given in the lower part of Table II and are representative of similar results, obtained with blood from the others. All the data obtained with sera from patients with malaria may be summarized as follows: From three of the patients, serum was gotten before treatment was begun. The outcome of the tests was similar to that obtained with normal serum, indicating that the chronic syphilitic infection did not evoke the serological response of acute infections. From two of the patients, the second sample of blood was acquired within 24 to 48 hours of the first rise in temperature. Streptococcidal action was demonstrable with both of the specimens. Subsequent bleedings were made both at times of highest fever and during temporary remissions of 1 to 3 days. Under both conditions, the capacity to destroy streptococci was present in the sera, but the technique which was used did not reveal any consistent quantitative differences. However, when the infection was terminated by quinine, within the following 2 to 3 days the activity of the specimens of the patients' sera returned to the normal level of the pre-infection sample. In these few observations, therefore, it appears that the streptococcidal element is maintained in the blood of patients with malaria regardless of the time in the swings of fever at which the blood is taken. With the permanent loss of fever, however, the blood rapidly returns to normal.

A comparison of the results obtained with sera from cases of pneumonia with that derived from cases of malaria suggests that the former specimens were more potent in streptococcidal power. This is of interest in view of the fact that the duration of active infection in pneumonia was often not as prolonged as in malaria and also that the fever in pneumonia, although usually continuous instead of intermittent, did not regularly reach the height exhibited by the malarial patients. 
Although the methods which were employed afforded only a rough quantitative measure of streptococcidal activity, the impression has been gained that sera of greatest killing power came from individuals whose illness was most severe.

\section{DISCUSSION}

The observations which have been recorded demonstrate the striking capacity of sera from patients with acute infections to destroy hemolytic streptococci. Under the experimental conditions employed, the rapidity with which the number of viable organisms introduced into the serum was found to diminish and, finally, to disappear completely, is indicative of the potency of the serological property responsible for the bactericidal effect. By making repeated observations on successive samples of serum from the same individual it was found that recovery of the patient was followed by a great decrease in killing action of the blood serum. With blood from patients whose recovery was abrupt, as in pneumonia terminating by crisis or in malaria responsive to quinine, the rapid loss of the streptococcidal power of the serum following the cessation of active disease was very striking. When improvement was slower, the decrease in the serological property was also delayed. The results indicate that when the stimulus of active infection was overcome, the body factors, whatever their nature, which are responsible for the continued presence in the blood of the bactericidal property, also ceased to operate.

From the tables it may be noted that there is a relatively close parallelism between the bactericidal capacity of the serum and the temperature of the patient at the time blood was withdrawn. It will be of interest to attempt to determine whether fever itself is of prime importance in influencing changes in the blood serum or whether other factors inherent in active febrile disease cause the appearance and disappearance of the humoral bactericidal property.

The time relationship between the presence or absence of the streptococcidal property and the course of the disease, which has just been discussed, exemplifies a difference between this particular antibacterial activity and the well defined antibodies reactive with bacteria such as agglutinins. Furthermore, the fact that sera from patients having pneumococcus, hemolytic streptococcus, staphylo- 
coccus, meningococcus, or malarial infections, all possess the streptococcidal property, is an additional observation which differentiates this reaction from the specific antibacterial response which so commonly follows infections.

The differences in the susceptibility of the three strains of hemolytic streptococci which were employed in this study, are also of interest. One strain $(\mathrm{Sc})$ was destroyed by sera from all of the acutely ill patients. On the other hand, the viability of another strain $(\mathrm{Ba})$ was affected by only a few sera. The third strain (Co) possessed a degree of sensitiveness intermediate between the other two. The results obtained with the three separate strains were used as a rough measure of the streptococcidal potency of the sera. Specimens able to act upon all three strains were considered to be the most powerful; those affecting strains Co and Sc were deemed to be somewhat less so; and finally, the sera capable of killing only strain Sc were classified as the least potent. Proof of the correctness of this assumption must await further investigation with a technique which will afford more accurate quantitative measurements. However, it should be emphasized that differences in the behavior of strains need to be taken into consideration in performing experiments such as those which have been described.

\section{SUMMARY}

Sera obtained from patients at the time of acute active infections were found, in every instance, to be bactericidal for hemolytic streptococci. The observations were made with sera from twenty-five patients. The group consisted of cases of pneumococcus, hemolytic streptococcus, staphylococcus, meningococcus, tubercle bacillus, and malarial infections; the etiology of the diseases in other patients was either uncertain or may have been a mixed infection.

In sera taken from the same group of patients, soon after recovery or marked improvement had taken place, the streptococcidal property was absent or greatly diminished in potency.

By comparative tests made with sera from twenty healthy adults, the streptococcidal action was not demonstrable.

Three different strains of Streptococcus hemolyticus of the beta type 
were employed in the experiments. Differences in susceptibility of the strains to the killing power of the serum were noted.

\section{BIBLIOGRAPHY}

1. Tillett, W. S., J. Exp. Med., 1937, 65, 163.

2. Dresel, E. G., and Keller, W., Zentr. Bakt., 1. Abi., Orig., 1922-23, 89, suppl., 240.

3. Trommsdorff, R., Zentr. Bakt., 1. Abt., Orig., 1902, 32, 439.

4. Knorr, M., Baktericide und Bakteriolytische Stoffe des Blutes, in Kolle, W., and von Wassermann, A., Handbuch der pathogenen Mikroorganismen, Jena, Gustav Fischer, 3rd edition, (Kolle, W., Kraus, R., and Uhlenhuth, P.) 1929, 2, 663.

5. Topley, W. W. C., and Wilson, G. S., The principles of bacteriology and immunity, London, Edward Arnold \& Co., 1929, 1, 169. 